\title{
Dealing with immune-mediated neuropathies during COVID-19 outbreak: practical recommendations from the task force of the Italian Society of Neurology (SIN), the Italian Society of Clinical Neurophysiology (SINC) and the Italian Peripheral Nervous System Association (ASNP)
}

\author{
Raffaele Dubbioso ${ }^{1}$ (D) Eduardo Nobile-Orazio ${ }^{2} \cdot$ Fiore Manganelli ${ }^{1} \cdot$ Lucio Santoro $^{1} \cdot$ Chiara Briani $^{3}$ - Dario Cocito ${ }^{4}$. \\ Gioacchino Tedeschi ${ }^{5}$. Vincenzo Di Lazzaro ${ }^{6}$ - Gian Maria Fabrizi ${ }^{7}$ on behalf of SIN, SINC and ASNP
}

(C) Fondazione Società Italiana di Neurologia 2020

\section{Introduction}

Since December 2019 when the novel coronavirus, currently named severe acute respiratory syndrome coronavirus 2 (SARS-CoV-2), outbreak has been described in Wuhan, Hubei region, China, the situation has dramatically evolved [1-4]. The World Health Organization (WHO) has recently declared coronavirus disease 2019 (COVID-19) a public health emergency of international concern [5]. The pandemic, as declared by the $\mathrm{WHO}$, has led to $>2.000 .000$ cases and $>130.000$ COVID-19-related deaths worldwide reported as of April 2020, in all continents spreading on a logarithmic scale in Europe and the USA.

Italy is currently one of the most affected countries together with the USA, Spain, France and Germany. There are no known proven therapies for treating this virus and no vaccine to prevent the infection at this time.

Raffaele Dubbioso and Eduardo Nobile-Orazio contributed equally to this work.

Raffaele Dubbioso

rafdubbioso@gmail.com

Fiore Manganelli

fioremanganelli@gmail.com

1 Department of Neurosciences, Reproductive Sciences and Odontostomatology, University of Naples "Federico II", Via S. Pansini, 5, IT-80131 Naples, Italy

2 Department of Medical Biotechnology and Translational Medicine, Neuromuscular and Neuroimmunology Service, Humanitas Clinical and Research Institute, Milan University, Via Manzoni 56, Rozzano, Milan, Italy
As the rate of hospitalization is very high among symptomatic cases, with an increased need to have access to intensive care units (ICUs) and mortality in the order of 3\% globally [3], European hospitals have been forced to intensively reduce elective activities, including outpatient activity, in order to face the high numbers of admissions. In addition, action by governments to contain the outbreak and slowdown the spread of COVID-19 has restricted regions and nations by reducing their mobility within countries and across borders.

Symptoms of COVID-19 are variable but typically include fever, cough, respiratory symptoms and diarrhoea. Severity ranges from mild to severe and the virus may lead to pneumonia, acute respiratory distress syndrome and death. It has been reported that more than one-third of patients experienced various neurological symptoms including the involvement of central nervous system (i.e. dizziness, headache, impaired consciousness, ataxia and epilepsy), peripheral nervous

3 Department of Neurosciences, University of Padova, Padova, Italy

4 Istituti Clinici Scientifici Maugeri, Torino, Italy

5 Division of Neurology and Neurophysiopathology, Department of Advanced Medical and Surgical Sciences, University of Campania "Luigi Vanvitelli", Naples, Italy

6 Neurology, Neurophysiology and Neurobiology Unit, Department of Medicine, Università Campus Bio-Medico di Roma, Rome, Italy

7 Section of Clinical Neurology, Department of Neurosciences, Biomedicine and Movement Sciences, University of Verona, University Hospital “G.B. Rossi”, P.le L.A. Scuro 10, 37134 Verona, Italy 
system (i.e. taste, smell and vision impairment and neuralgia) and skeletal muscular damage [6,7]. It is also frequent to find a concomitant COVID-19 infection in patients presenting with acute neurological disorders including stroke and seizure [7].

With regard to the peripheral nervous system, it is still unknown if and how SARS-CoV-2 can affect it in previously healthy individuals or in patients with a diagnosis of neuropathy. There are a few anecdotal reports of acute immunemediated neuropathy (i.e. Guillain-Barré and MillerFisher syndromes) in patients with coronavirus infection, including SARS-CoV-2 [8-10] and Middle East Respiratory Syndrome-CoV [11], raising the possibility that peripheral nervous system can be directly or indirectly affected by the virus. The main concern resides however for patients with peripheral nerve disorders on immunosuppressive or immunomodulatory therapies who may also have respiratory muscle weakness. These patients may be at higher risk of contracting the infection and/or of experiencing severe manifestations of COVID-19. There are numerous recommendations trying to provide clarity and guidance even if, so far, there are no formal evidence-based recommendations from clinical societies or governments for the management of immunosuppressive treatment in these patients. Immunotherapy decision varies significantly from country to country, ranging from highly provider directed to collaborative decision-making model. In order to inform the neurological community and patients, the Italian Association of the Peripheral Nervous System (ASNP), the Italian Society of Clinical Neurophysiology (SINC) and the Italian Society of Neurology (SIN) have developed a joint document to provide the best practices for managing patients with immune-mediated neuropathy during the global spread of COVID-19. The following document should be interpreted as a collection of indications or advice developed by neurologists with expertise in immune-mediated polyneuropathies (i.e. Guillain-Barré syndrome and its variants (GBS); chronic inflammatory demyelinating polyradiculoneuropathy (CIDP), multifocal motor neuropathy (MMN), Lewis-Sumner, polyneuropathies associated with monoclonal gammopathy with or without anti-MAG antibodies and neuropathies during vasculitis). These practical recommendations should be individualized according to the severity and the progression of the neuropathy, the local healthcare strategic planning and COVID-19 infection risk.

\section{Do patients with immune-mediated neuropathy have an increased risk of contracting SARS-CoV-2 infection?}

- To date, there is neither scientific evidence that immunemediated neuropathy itself increases the risk of SARSCoV-2 infection nor evidence of association between virus infection and the development of immune-mediated neuropathy.
- Outpatient visits and treatments should be possibly avoided or postponed, to avoid a possible contamination during transfer to the hospitals or within the hospital. Whenever possible, the in-person care should be converted to telemedicine visits or e-consultations in order to provide the regular follow-up of patients. However, the feasibility of virtual approach should be evaluated locally because at present there are several barriers to the implementation of telehealth.

- Immunosuppressive medications may increase susceptibility to infections, including SARS-CoV-2.

\section{What to do if a patient is on immunosuppressant drugs?}

- Patients should be informed that reducing or stopping an existing immunosuppressive therapy may lead to an increase of disease activity and/or to exacerbation of the neuropathy.

- It is strongly recommended that patients under treatment with first-line (i.e. steroid) and second-/third-line medications (i.e. azathioprine, methotrexate, cyclosporine, mycophenolate mofetil and cyclophosphamide orally) continue treatment with high attention to personal protective equipment. Beyond the recommendations of the Ministry of Health and National and Local laws valid for the whole population, additional advice for patients is to:

- avoid leaving the house for any reason including shopping;

- work from home whenever this is possible;

- go to the hospital only in exceptional cases, in any case not before having contacted the treating neurologist;

- entrust family members to pick up the treatment provided by the pharmacy or hospital.

- Patients on pulsed treatment with intravenous corticosteroids should continue therapy at home. If this is not possible, evaluate the possibility to perform the same therapy at home at the same dosage intramuscularly or orally with an equivalent dosage of another corticosteroid.

- If infusion therapy with rituximab or cyclophosphamide is planned in the hospital, it is recommended to evaluate the risk-benefit ratio of postponing the treatment.

- For maintenance rituximab therapy, it is suggested to delay the infusion even beyond 6 months, if the CD19 and CD20 lymphocytes are suppressed.

- If immunosuppressive treatment requires frequent blood monitoring, evaluate the possibility to reduce the frequency or, if possible, to perform home blood sampling. 
What to do if a patient is on immunoglobulin therapy?

- Treatment with intravenous (IVIG) or subcutaneous (SCIG) immunoglobulins does not increase the risk of contracting SARS-CoV-2 infection. Immunoglobulins do not suppress the immune response but rather enhance it. There is however, no evidence so far that immunoglobulins may be protective against SARS-CoV-2.

- Patients under treatment with IVIG may need to go frequently to the Hospital or infusion centres and are therefore exposed to a potential increased risk of infection. Taking into account also the regional variability of COVID-19 risk, it is advisable to discuss with the patient the possibility switch the treatment to subcutaneous immunoglobulins (SCIG).

\section{What to do if a patient is treated with plasmapheresis?}

- Treatment with plasmapheresis does not increase the risk of getting SARS-CoV-2 infection.

- To perform plasmapheresis, however, patients must go to the hospital and therefore may be exposed to a potential greater risk of infection.

\section{How to monitor patients with immune-mediated neuropathy?}

- At this time, it is strongly discouraged to request clinical and instrumental evaluations to monitor the disease course (e.g. blood tests, electroneurography/myography, nerve ultrasound, MRI) unless considered essential by the neurologist.

- We strongly recommend to continue to monitor and assess the patients by telephone call or telemedicine (e.g. via Skype) in order to avoid that the patients move from a protected place (home) to a place theoretically at a greater risk of infection (hospital). The validity of telemedicine in assessing patients with polyneuropathy has been recently documented [12].

\section{Can I start treatment in a patient with immune-mediated neuropathy?}

- At this time, unless clinical conditions make it necessary, it is recommended not to start any treatment with immunosuppressive or immunomodulatory medications thus avoiding to suppress the immune system as well as to expose patients to risk of infections related to access to the hospital for therapy or analysis laboratories (blood tests are usually carried out before starting therapy).

- Concerning immunoglobulin therapy in non-acute forms of disease, the risk associated with repeated access to the hospital or infusion centres should be evaluated. When indicated, steroid treatment may be preferred even if it may reduce the immune response.

\section{What to do if a patient with immune-mediated neuropathy is positive for SARS-CoV-2?}

- Currently, there is no evidence to support that the discontinuation of immunosuppressive treatment may be associated with a better prognosis:

- Data from previous coronavirus epidemics (SARS and MERS) suggest that immunocompromised patients are not exposed to more aggressive lung disease;

- It is however advisable to postpone therapy with rituximab and cyclophosphamide during the positivity phase for SARS-CoV-2 infection or during the quarantine period;

- Stopping or reducing therapy should be agreed with treating neurologist;

- Steroid therapy should be not suspended abruptly but should be progressively reduced until suspension;

- There is no contraindication to immunoglobulin treatment. However, since IVIG may be associated to a possible increase in risk of venous thrombosis, it should not be overlooked that patients with COVID-19 are at high risk for venous thromboembolism. It is unknown whether immunoglobulins may have any protective role.

- During the positivity phase for SARS-CoV-2 infection or during the quarantine period, the initiation of any immunosuppressive therapy it is not recommended.

\section{Special precautions in performing neurophysiologic testing}

Neurophysiological assessment, including nerve conduction studies and electromyography, could be required for diagnostic work-up in patients with suspected diagnosis of immunemediated neuropathy and especially for those admitted to intensive care units (ICUs). 
Neurophysiological investigations require close contact with the patient and may result in an increased risk of reciprocal transmission of the infection. Neurophysiological testing should be therefore only requested if it might substantially change the management of the patients. All other unnecessary neurophysiological examination should be therefore postponed.

Physicians and technicians should use standard individual protection devices and should also provide surgery masks and gloves to patients not suspected to have SARS-CoV-2 infection. For COVID-19 patients, physicians and technicians should wear personal protective equipment (PPE) including appropriate masks, face shields, gowns and gloves.

In addition, disposable materials should be used and special attention should be paid in cleaning the equipment.

However, all the decisions should be managed according to local policies and guidelines. For further information, the American Association of Clinical Neurophysiology website has guidance (https://www.acns.org/practice/covid-19resources).

\section{Concluding remarks}

Immune-mediated neuropathies are not associated with an increased risk for an unfavourable outcome of COVID-19. Immunosuppressive therapies as well as treatment of the patient in the hospital as inpatient or outpatient might increase the risk of infection.

Treatment should be individualized balancing the severity of neuropathy, its progression and the risk of getting infected in specific geographic area.

Countries have to face the possibility that individual case containment might not be possible in the near future. It is however important that the re-introduction of adequate management and care of patients with immune-mediated neuropathy and the resumption of vital clinical and preclinical research will be possible during the control of the COVID-19 outbreak.

This global crisis may however contribute to change significantly the care of our patients toward a better acceptance of virtual consultations and assessments by telemedicine.

We continue to monitor this rapidly evolving situation and we will update our recommendations whenever new data become available.

\section{Compliance with ethical standards}

Conflict of interest The authors declare that they have no conflict of interest.

Ethical approval None.

\section{References}

1. Guan W-J, Ni Z-Y, Hu Y, Liang WH, Ou CQ, He JX, Liu L, Shan H, Lei CL, Hui DSC, du B, Li LJ, Zeng G, Yuen KY, Chen RC, Tang CL, Wang T, Chen PY, Xiang J, Li SY, Wang JL, Liang ZJ, Peng YX, Wei L, Liu Y, Hu YH, Peng P, Wang JM, Liu JY, Chen Z, Li G, Zheng ZJ, Qiu SQ, Luo J, Ye CJ, Zhu SY, Zhong NS (2020) Clinical characteristics of coronavirus disease 2019 in China. N Engl J Med [Epub ahead of print]. https://doi.org/10.1056/ NEJMoa2002032

2. Chen T, Wu D, Chen H, Yan W, Yang D, Chen G, Ma K, Xu D, Yu H, Wang H, Wang T, Guo W, Chen J, Ding C, Zhang X, Huang J, Han M, Li S, Luo X, Zhao J, Ning Q (2020) Clinical characteristics of 113 deceased patients with coronavirus disease 2019: retrospective study. BMJ 368:m1091. https://doi.org/10.1136/bmj.m1091

3. Huang C, Wang Y, Li X, Ren L, Zhao J, Hu Y, Zhang L, Fan G, Xu J, Gu X, Cheng Z, Yu T, Xia J, Wei Y, Wu W, Xie X, Yin W, Li H, Liu M, Xiao Y, Gao H, Guo L, Xie J, Wang G, Jiang R, Gao Z, Jin Q, Wang J, Cao B (2020) Clinical features of patients infected with 2019 novel coronavirus in Wuhan, China. Lancet 395:497-506. https://doi.org/10.1016/S0140-6736(20)30183-5

4. Wang D, Hu B, Hu C, Zhu F, Liu X, Zhang J, Wang B, Xiang H, Cheng Z, Xiong Y, Zhao Y, Li Y, Wang X, Peng Z (2020) Clinical characteristics of 138 hospitalized patients with 2019 novel coronavirus-infected pneumonia in Wuhan, China. JAMA - J Am Med Assoc 323:1061-1069. https://doi.org/10.1001/jama.2020. 1585

5. World Health Organization Q\&A on coronaviruses (COVID-19) (2020) Available on https://www.who.int/news-room/q-a-detail/qa-coronaviruses

6. Jin $\mathrm{H}$, Hong $\mathrm{C}$, Chen $\mathrm{S}$ et al (2020) Consensus for prevention and management of coronavirus disease 2019 (COVID-19) for neurologists. Stroke Vasc Neurol 2019:svn-2020-000382. https://doi.org/ 10.1136/svn-2020-000382

7. Mao L, Jin H, Wang M, Hu Y, Chen S, He Q, Chang J, Hong C, Zhou Y, Wang D, Miao X, Li Y, Hu B (2020) Neurologic manifestations of hospitalized patients with coronavirus disease 2019 in Wuhan, China. JAMA Neurol. https://doi.org/10.1001/ jamaneurol.2020.1127

8. Zhao H, Shen D, Zhou H, Liu J, Chen S (2020) Guillain-Barré syndrome associated with SARS-CoV-2 infection: causality or coincidence? Lancet Neurol S1474-4422:30109-30105. https://doi. org/10.1016/S1474-4422(20)30109-5

9. Gutiérrez-Ortiz C, Méndez A, Rodrigo-Rey S et al (2020) Miller fisher syndrome and polyneuritis cranialis in COVID-19. Neurol 241. https://doi.org/10.1212/WNL.0000000000009619

10. Toscano G, Palmerini F, Ravaglia S et al (2020) Guillain-Barré syndrome associated with SARS-CoV-2. N Engl J Med. https:// doi.org/10.1056/NEJMc2009191

11. Kim JE, Heo JH, Kim HO, Song SH, Park SS, Park TH, Ahn JY, Kim MK, Choi JP (2017) Neurological complications during treatment of middle east respiratory syndrome. J Clin Neurol 13:227233. https://doi.org/10.3988/jen.2017.13.3.227

12. Wilson AM, Jamal NI, Cheng EM, Inkelas M, Saliba D, Hanssen A, Torres JA, Ong MK (2020) Teleneurology clinics for polyneuropathy: a pilot study. J Neurol 267:479-490. https://doi. org/10.1007/s00415-019-09553-0

Publisher's note Springer Nature remains neutral with regard to jurisdictional claims in published maps and institutional affiliations. 